\title{
Incidental Discovery of Emphysematous Cystitis with Rapid Resolution in a Patient Presenting with Acute Pancreatitis
}

\author{
M. Al-Assiri* and P. Chan \\ Department of Urology, Royal Victoria Hospital, McGill University Health Centre, \\ Montreal, Quebec, Canada \\ E-mail: manaalassiri@yahoo.com \\ Received July 26, 2004; Revised August 27, 2004; Accepted August 30, 2004; Published September 30, 2004
}

KEYWORDS: cystitis,bladder disease,emphysema,pancreatitis

DOMAIN: urology

\section{CASE HISTORY}

A 58-year-old male presented with a 2-h history of epigastric pain, nausea, and vomiting after an episode of heavy alcohol consumption. He denied any irritative and obstructive urinary symptoms.

On physical examination:

1. Afebrile with stable vital signs.

2. Abdominal examination revealed epigastric tenderness with rebound.

Investigation:

1. Elevated lipase level (90 U/L), his serum biochemical profile was essentially normal.

2. A urinalysis was positive for red blood cells, negative for white blood cells and nitrites.

A clinical diagnosis of acute pancreatitis was made.

A computerized tomography of the abdomen and pelvis was consistent with a Grade A pancreatitis (Fig. 1). Incidentally, air was noted within the urinary bladder wall (Fig. 2). Of note, there was no recent urinary tract instrumentation.

The diagnosis was emphysematous cystitis.

The patient was treated with oral antibiotics with prompt clinical response. Three days later, a followup CT abdomen and pelvis showed significant resolution of the emphysema in urinary bladder (Fig. 3). No significant change was noted in appearance of the pancreas (Fig. 4).

This case shows that a life-threatening urological condition could present with a nonurological complaint. 


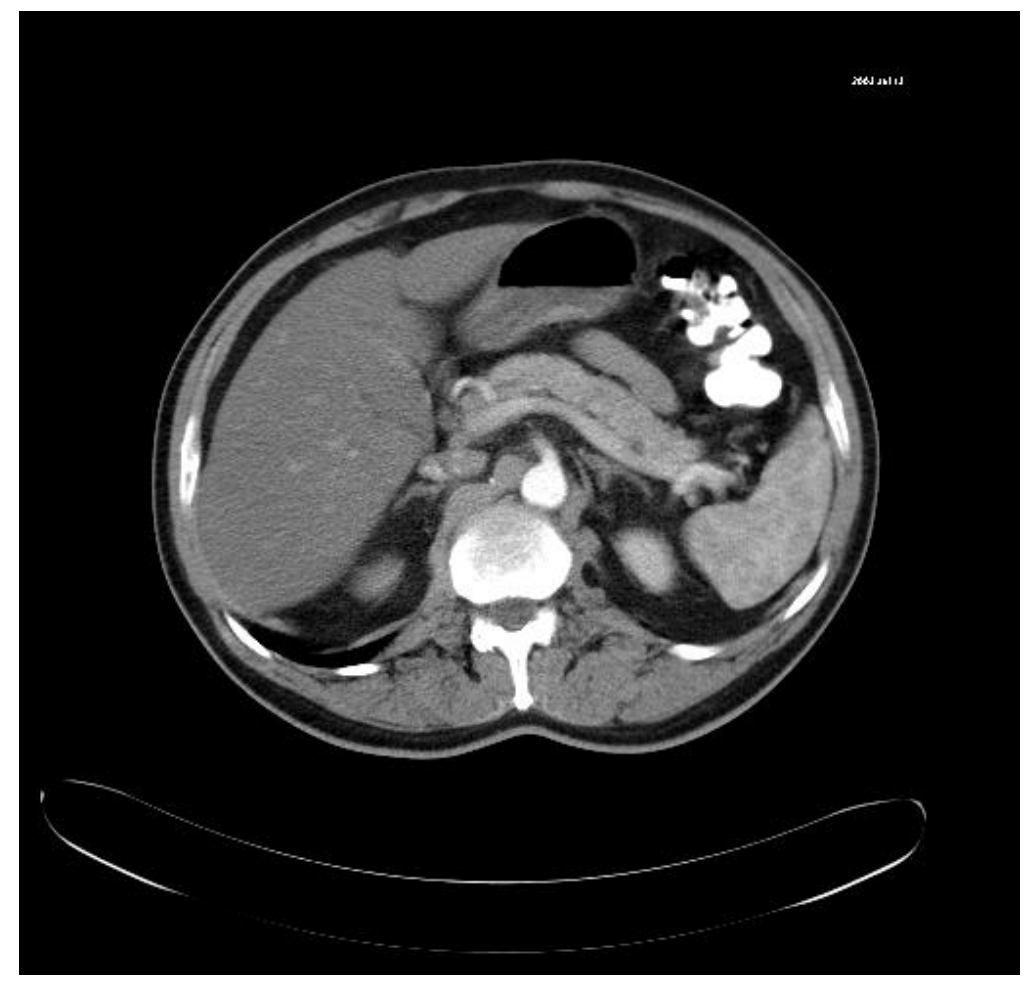

FIGURE 1. CT scan of the upper abdomen and pelvic demonstrating Grade A pancreatitis.

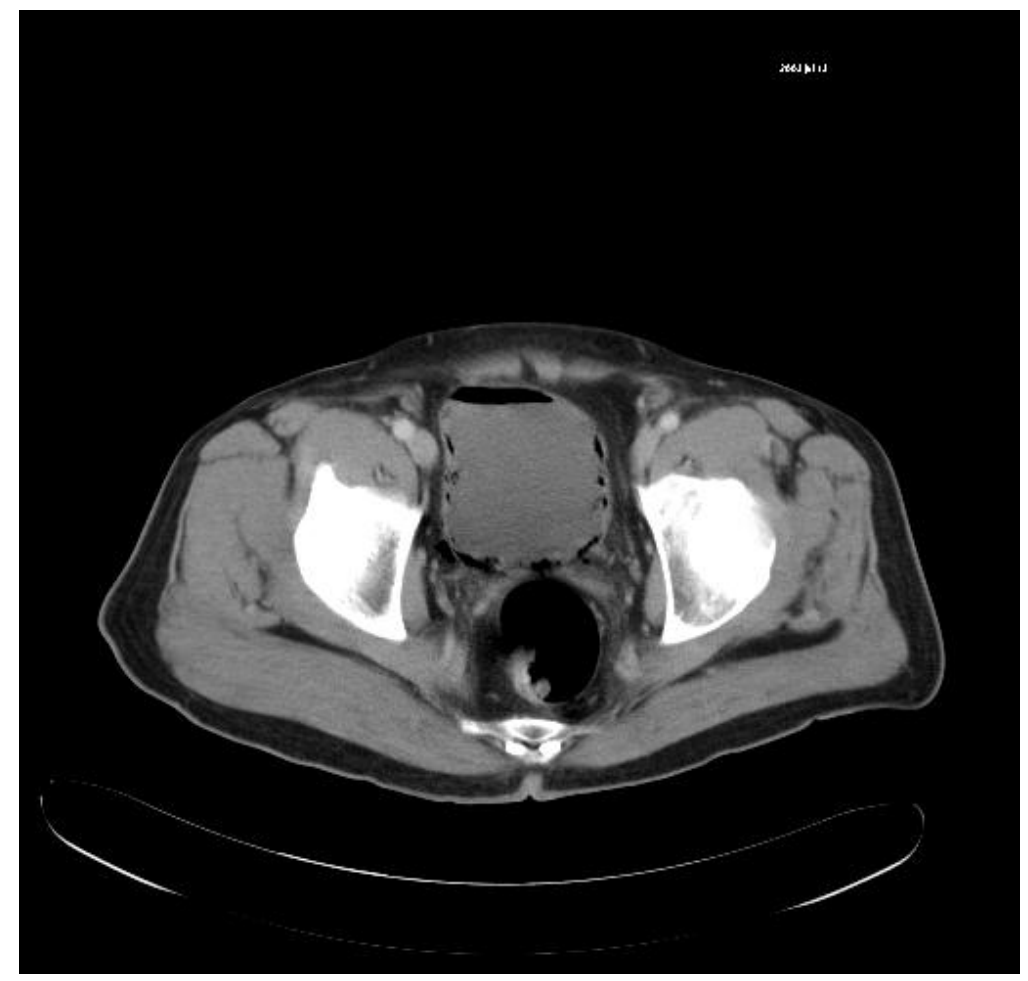

FIGURE 2. Intramural and intraluminal gas in the urinary bladder. 


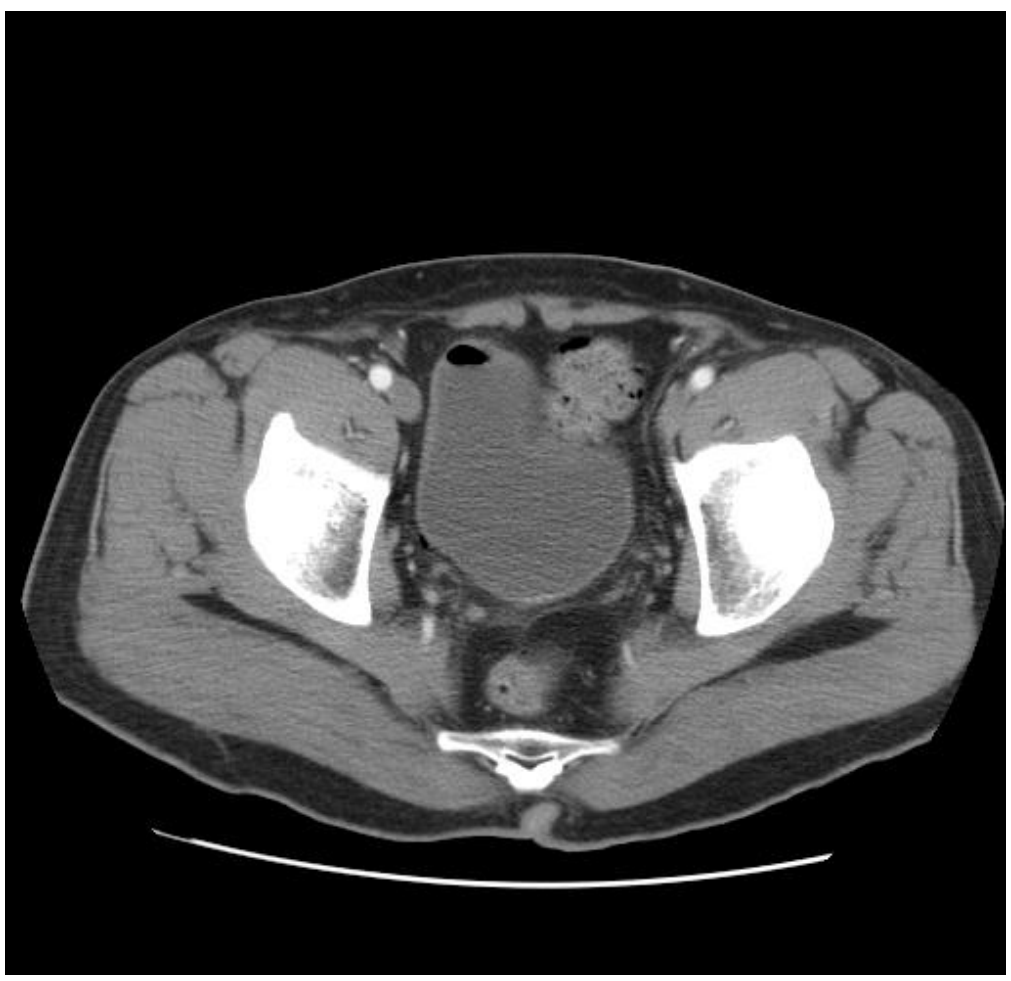

FIGURE 3. A follow-up CT obtained 3 days later demonstrated nearly a complete resolution of the intramural and intraluminal gas in the urinary bladder.

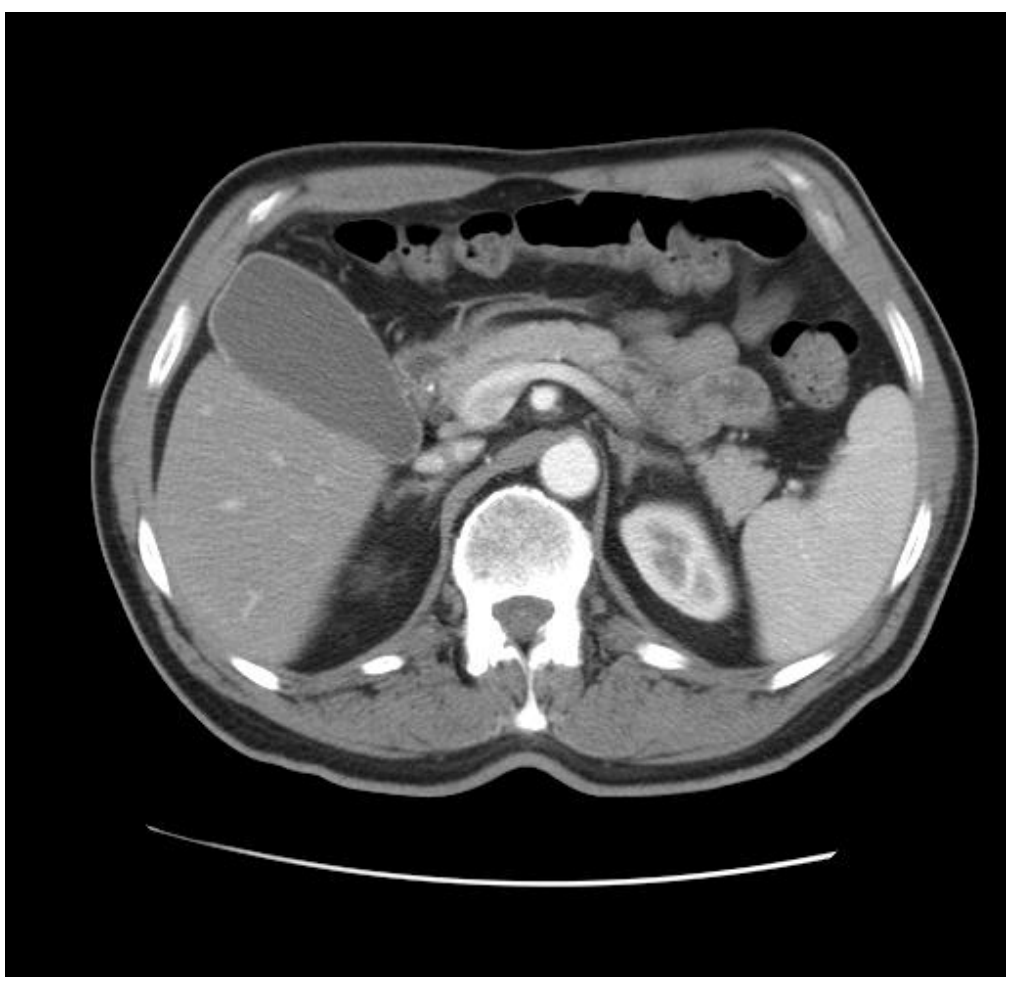

FIGURE 4. No significant change was observed in the appearance of the pancreas. 

cystitis

\section{This article should be referenced as follows:}

Al-Assiri, M. and Chan, P. (2004) Incidental discovery of emphysematous cystitis with rapid resolution in a patient presenting with acute pancreatitis. TheScientificWorldJOURNAL 4, 881-884.

\section{Handling Editor:}

Anthony Atala, Principal Editor for Urology — a domain of TheScientificWorldJOURNAL. 


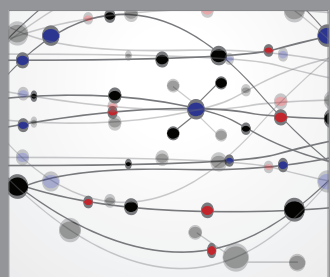

The Scientific World Journal
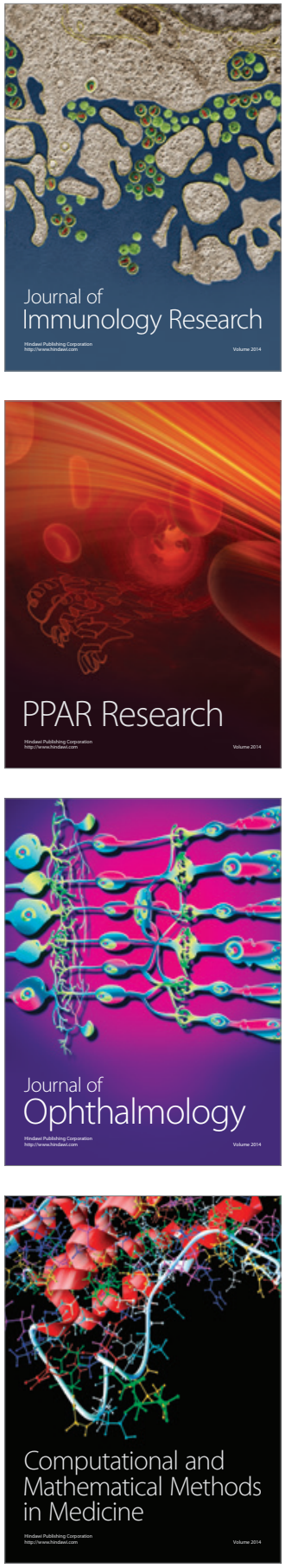

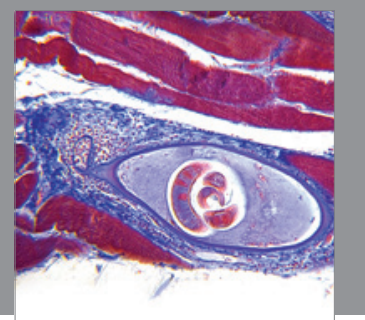

Gastroenterology

Research and Practice
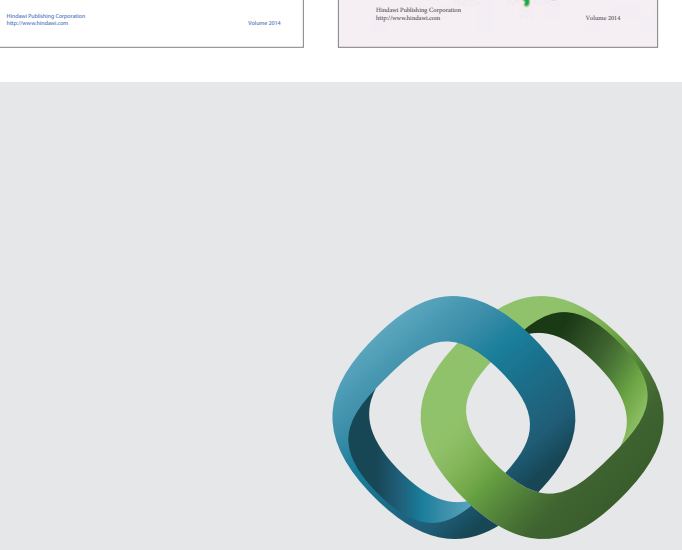

\section{Hindawi}

Submit your manuscripts at

http://www.hindawi.com
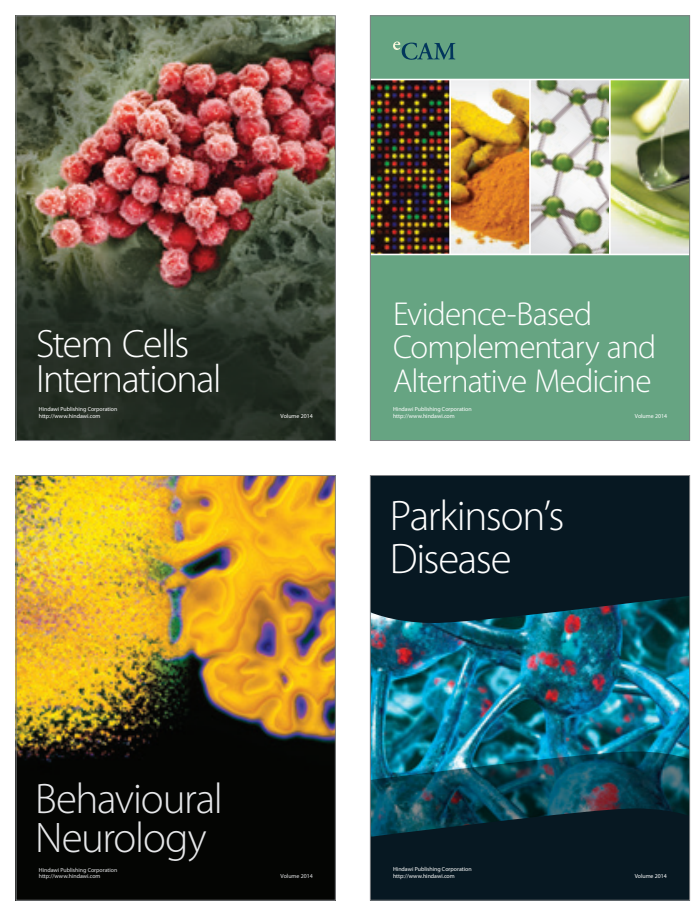

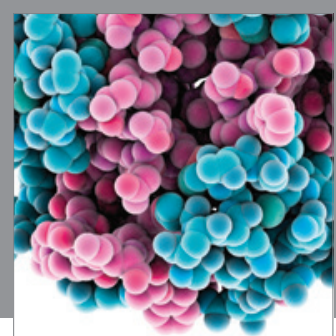

Journal of
Diabetes Research

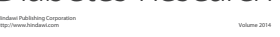

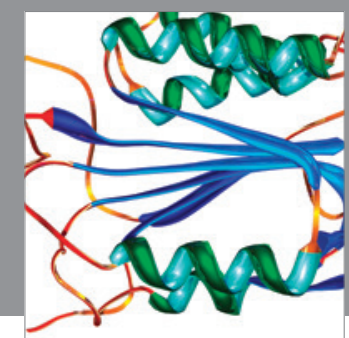

Disease Markers
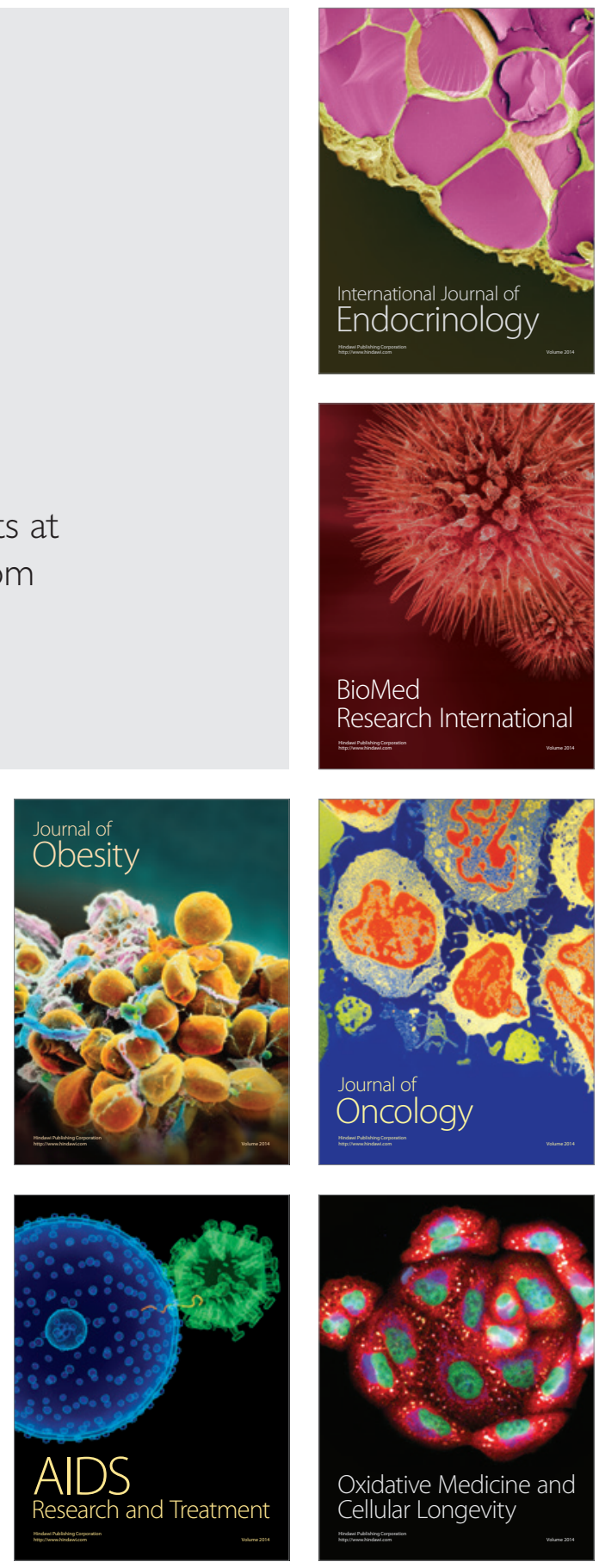